\title{
BENDO-TENSEGRITY MODEL SIMULATES COMPRESSION TEST OF ANIMAL CELL
}

\author{
Y. D. Bansod", Veera V. S. V. P. Jakka ${ }^{* *}$, J. Burša ${ }^{* * *}$
}

\begin{abstract}
A hybrid model of suspended animal cell proposed earlier, with a bendo-tensegrity structure mimicking cytoskeleton, is applied to simulate the global response of the cell under compression and to describe mechanical behaviour of its components. The Finite Element model incorporates Microtubules, Actin Filaments, Intermediate Filaments, nucleus, cytoplasm, and Cell Membrane, all of them with realistic geometrical and material parameters. The unique features of this structural model keep fundamental principles governing cell behaviour, such as interaction between the cytoskeletal components redistributing the prestress of actin filaments throughout all the structure. The force-deformation curve from the simulated compression test with microplates is validated by comparison with the experimental response from literature. The model enables us to investigate the mechanical role of individual celular and cytoskeletal components in intracellular force propagation by means of changing their numbers or parameters. As quantitative characterization of nucleus deformation may be hypothetically decisive for mechanotransduction, the model aims at better understanding of how cellular processes are mechanically controlled.
\end{abstract}

Key words: cell mechanics, cytoskeleton, bendo-tensegrity model, compression test simulation

\section{Introduction}

Mechanical testing of individual cells represents an important source of input data for modelling of their mechanical behaviour. Simulations of these tests enable identification of the models and aim at explaining cellular processes such as mechanotransduction. They represent also a step needed in multilevel modelling of tissues. Different types of cell models have been created using continuum and discrete approaches, or combination of both in hybrid models. Tensegrity models represent one of the most promissing concepts, showing however some limitations (see Bursa et al., 2012) which have been overcome using bendotensegrity concept. Such hybride model was created in (Bansod, 2016) using finite element program system ANSYS (ANSYS Inc. PA, US); it encompasses the cell nucleus and cytoplasm surrounded by the Cell Membrane (CM), as well as cytoskeletal components like Actin Filaments (AFs), Micro Tubules (MTs), and Intermediate Filaments (IFs). Microscopic observations of the cell shape in suspended state as well as images of distributions of cytoskeletal proteins were referred to create the 3D suspended cell model and the architecture of its cytoskeletal components. In constrast to the tensegrity models published earlier, MTs are modelled here more realistically as bended (buckled) beams reducing thus their stiffness under compression and making their response under tension more non-linear (Mehrbod et al., 2011). This paper presents results of simulations of compression test with microplates of an isolated suspended cell using this hybrid model.

\section{Methodology}

The proposed model implemented the hybrid modelling approach, i.e. the continuum parts (nucleus, cytoplasm) were modelled using continuous (volume) finite elements circumscribed by a thin layer of shell elements representing CM, while the cytoskeletal components were modelled using discrete (beam

\footnotetext{
Ing. Yogesh Deepak Bansod, PhD.: Brno University of Technology, Brno; CZ, yogeshbansod@gmail.com

** Ing. Veera Venkata Satya Varaprasad Jakka, MSc.: Brno University of Technology, CZ, veeravenkata.jakka@mail.polimi.it

*** Prof. Ing. Jiř́i Burša, PhD.: Brno University of Technology, Brno; CZ, bursa@fme.vutbr.cz
} 
or truss) elements. The shape of the suspended cell model was defined as spherical with diameter $(D)$ of $32.2 \mu \mathrm{m}$, taken from one of the experimental measurements in (Nagayama et al., 2006). The nucleus was also modelled as spherical and positioned at the center of the cell. Similarly to a real cell, its position was stabilized by a network of IFs. Both cytoplasm and nucleus were modelled with eight-node hexahedral isoparametric elements; the flexible $\mathrm{CM}$ on the outer surface of cytoplasm was modelled by shell elements, with the thickness of $0.01 \mu \mathrm{m}$ (Rand, 1964) and no bending stiffness. The wavy IFs were modelled with negligible stiffness until being sufficiently (by $20 \%$ in the presented model) extended, while AFs created a network beneath the CM prestressed by means of their $-24 \%$ prestrain; both of them were modelled as tension-only truss elements. In contrast, MTs were modelled as bended beam structures, all of them originating from one point near the nucleus representing the centrosome. Geometrical as well as material parameters of all these components were set according to literature sources (see Bansod (2016) for more details). The contact between the cell and the rigid microplates was set as frictionless.

During the simulation, the cell model was compressed against the fixed (bottom) microplate by applying vertical displacements to the nodes of the movable (top) microplate to achieve $50 \%$ deformation of the cell. The reaction force was evaluated as the sum of forces at nodes of the contact surface between the cell and the movable microplate.

(a)

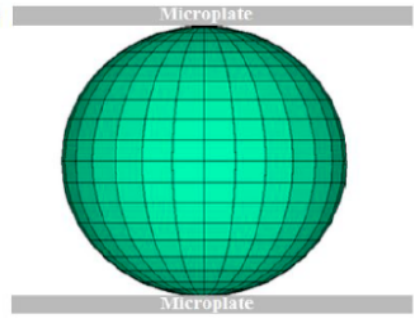

(c)

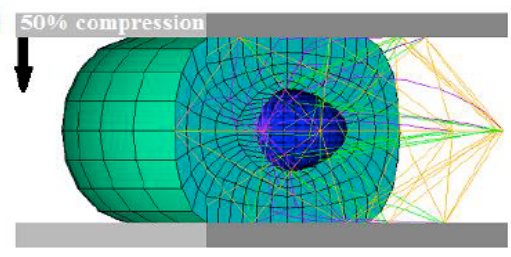

(b)

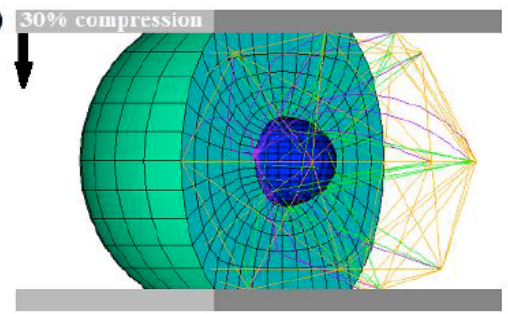

(d)

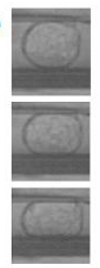

Fig. 1: Sectional views of the suspended cell model during consecutive steps in simulation of compression test: (a) spherical cell and microplates, compressing the cell with the movable microplate to (b) $30 \%$ and

(c) 50\% deformation against the fixed microplate. (d) Snapshots of a cell under compression in the corresponding phases of the experiment (Ujihara et al., 2012).

\section{Results and discussion}

The undeformed model and sectional views of some deformed shapes are presented in Fig. 1 in comparison with the snapshots from experiments. As depicted in Fig. 2, the force-deformation curve calculated from simulation is in good agreement with the non-linear response of the experimental results and validates thus partially the proposed bendo-tensegrity model. The slope of the simulated forcedeformation curve increases with increase in cell compression, similar to the effect observed in the experiments (Nguyen et al., 2009; Ujihara et al., 2012).

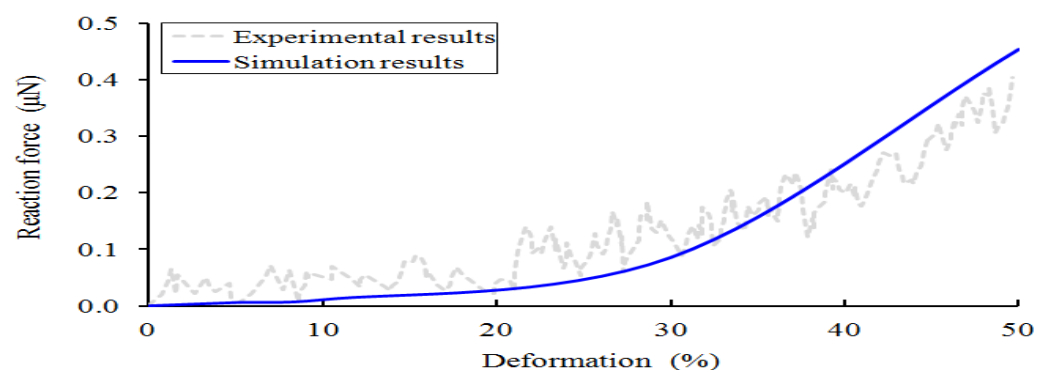

Fig. 2: Comparison of simulated force-deformation curve with the experimental dependence taken from a study by (Nguyen et al., 2009), investigating the biomechanical properties of a single chondrocyte using a micromanipulation technique. 
The compression stiffness of the model was calculated as the ratio of conventional stress to conventional strain. Here the reaction force of the cell at the compressed edge was $0.454 \mu \mathrm{N}$ and its (maximal undeformed) cross-sectional area $817 \mu^{2}$. The undeformed and deformed lengths of the cell corresponded to its full and half diameter, respectively, resulting thus in $50 \%$ compression and the respective stiffness of the model of $1.11 \mathrm{kPa}$.

\subsection{Response of individual cell components}

In addition to the global deformation presented in the above figures, the model enables to analyze also responses of the individual cell components under compression. Fig. 3(a) shows the deformed shape of the cell and nucleus; although they seem to be rather similar at the first glance, their aspect ratio (transversal to longitudinal dimension of the originally spherical shape) is very different: 2.1 for the cell against 1.3 for its nucleus. For the IFs the axial strain is chosen instead of stress as representative quantity because stress is zero up to the strain value set in the model to represent the waviness of IFs. The approximately ellipsoidal deformed shape of the nucleus appears analogous to that observed in experiments (Ujihara et al., 2012; Caille et al., 2002). During cell compression, the randomly oriented AFs and IFs were likely to be oriented perpendicularly to the loading direction; the filaments aligned in the loading direction were compressed whereas the perpendicular ones were stretched. The first principle strain in the nucleus is hypothesized to be the decisive quantity of mechanotransduction (Bursa et al., 2012).

The MTs localized in the central region perpendicularly to the direction of loading were straightened and became thus much stiffer than the bended ones. This effect, together with the AFs reoriented perpendicularly to the loading direction and resisting high tensile forces, contributes to the gradually increasing cell stiffness which corresponds to experimental observations (Ujihara et al., 2012). Low contribution of IFs to the cell response was detected because their negative as well as positive strains remained below their applied prestrain keeping thus their waviness and consecutively zero stresses.

\subsection{Analysis of mechanical contribution of the cell components}

The role of each cytoskeletal component in resisting cell compression (to $50 \%$ length) was investigated in greater detail via removal of each cytoskeletal component (individually as well as in all mutual combinations) from the above model which is denoted as control model in Fig. 4 and below. The maximum reaction force decreased by $26 \%$ without cytoskeleton with $20 \%$ attributed to AFs being thus the mechanically most significant cytoskeletal component.

Fig. 5 shows the influence of changes in the number of individual cytoskeletal components and in the CM thickness on the cell response under compression. Again, additional AFs created in the cell interior (together with additional nodes on the cell surface representing focal adhesions) increased the global cell reaction force, whereas increase in the density of either MTs or IFs did not show much variation. The

(a) Deformed shape

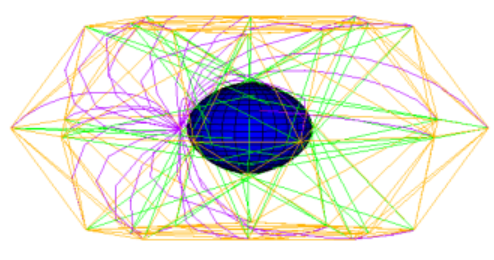

(d) Intermediate filaments $(I F \mathrm{~s})$

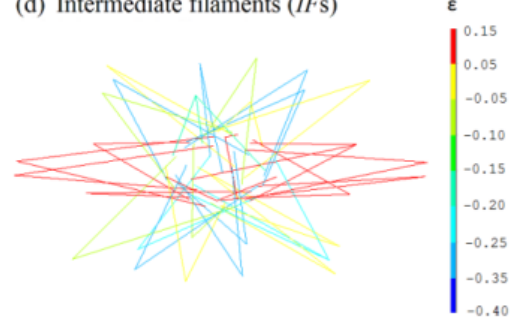

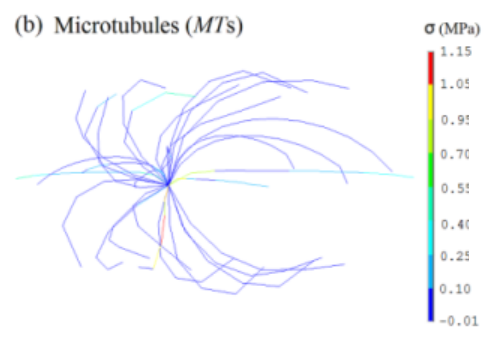

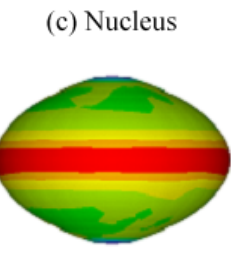

(e) Actin filaments (AFs)

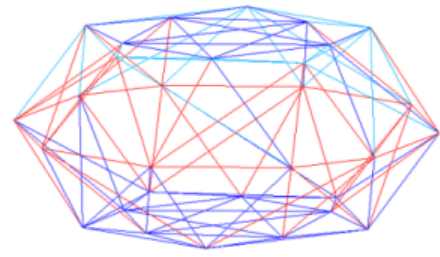

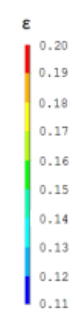

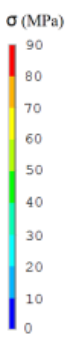

Fig. 3: Simulation results at 50\% cell compression: (a) deformed shape of the cell, cytoskeletal components and nucleus; distribution of axial stress in the (b) MTs (c) distribution of first principal strain in the nucleus; (d) distribution of axial strain in the discrete elements representing IFs; and (e) AFs; 


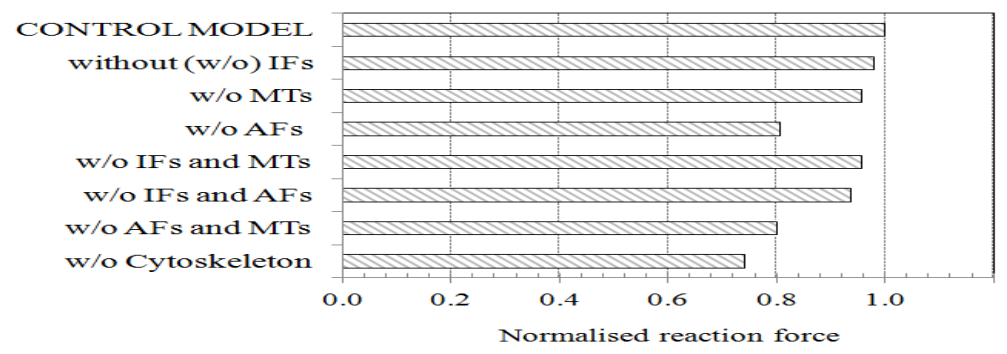

Fig. 4: Contribution of the cytoskeletal components individually and in mutual combinations to the cell stiffness at 50\% compression. The reaction force is normalized with respect to that of the control model

(a)

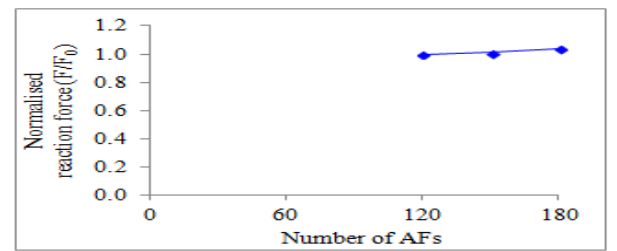

(c)

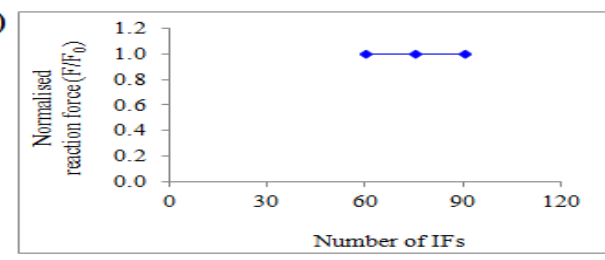

(b)

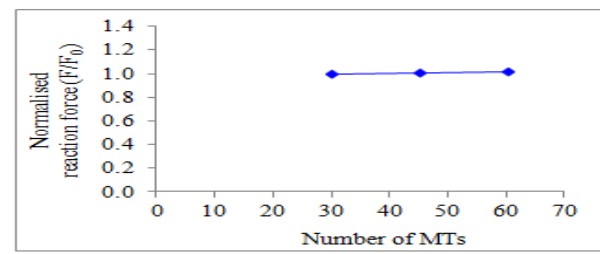

(d)

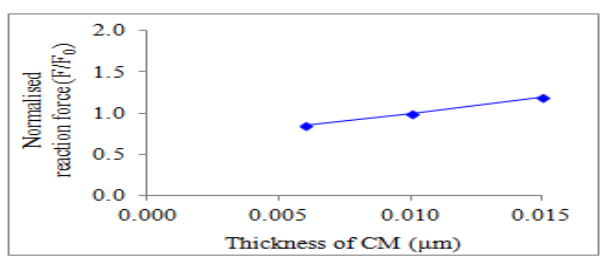

Fig. 5: Effect of increase in the density of cytoskeletal components (a) AFs, (b) MTs, and (c) IFs on the cell reaction force under 50\% compression; (d) similar effect of variation of CM thickness.

variations of $\mathrm{CM}$ thickness affected the overall cell reaction force most substantially. For the cytoskeletal components a similar effect could be achieved by a proportional increase of their elastic modulus or cross section area.

\section{Conclusion}

The proposed bendo-tensegrity model of a cell is capable to identify the influence of individual components on its global mechanical response, as well as the nucleus deformation, offering thus new insights into the interdependence of cellular mechanical properties and the mechanical role of cytoskeletal components. The compression test simulations revealed that although being tension-bearing components, actin filaments and cell membrane contributed most significantly to the cell response under compression.

\section{Acknowledgement}

This work was supported through NETME CENTRE PLUS (LO 1202) under the National Sustainability Programme I and by the Czech Science Foundation project no.18-13663S.

\section{References}

Bansod Y.D., (2016), Computational Simulations of Mechanical Tests of Isolated Animal Cells. Doctoral thesis, Brno University of Technology.

Bursa, J., Lebis, R., Holata, J, (2012), Tensegrity finite element models of mechanical tests of individual cells. Technology and Health Care, 20(2) (2012) 135-150.

Mehrbod, M., and Mofrad, R. K., (2011),On the significance of microtubule flexural behavior in cytoskeletal mechanics. PloS one, 6(10), e25627.

Nagayama, K., Nagano, Y., Sato, M., and Matsumoto, T., (2006). Effect of actin filament distribution on tensile properties of smooth muscle cells obtained from rat thoracic aortas. J Biomech, 39(2), 293-301.

Nguyen, B. V., Wang, Q., Kuiper, N. J., El Haj, A. J., Thomas, C. R., and Zhang, Z., (2009), Strain-dependent viscoelastic behaviour and rupture force of single chondrocytes and chondrons under compression. Biotechnology letters, 31(6), 803-9.

Rand, R., (1964), Mechanical properties of the red cell membrane: II. Viscoelastic breakdown of the membrane. Biophys J, 4(4), 303.

Ujihara, Y., Nakamura, M., Miyazaki, H., and Wada, S., (2012), Contribution of actin filaments to the global compressive properties of fibroblasts. Journal of the Mechanical Behavior of Biomedical Materials, 14, 192-8. 